\title{
CENTRAL LOCALIZATION AND GELFAND-KIRILLOV DIMENSION
}

BY

S. P. SMITH

ABSTRACT

Let $R$ be a factor ring of the enveloping algebra of a finite dimensional Lie algebra over a field $k$. If the centre of $R, Z$, consists of non-zero divisors in $R$, the ring $R_{Z}$ obtained by localizing at the non-zero elements of $Z$ becomes a finitely generated algebra over the field $K$ which arises as the field of fractions of $Z$. The Gelfand-Kirillov dimension of an $R$-module $M$ is denoted $d(M)$. In this paper it is shown that if $R_{Z} \otimes_{R} M \neq 0$ then $d(M) \geqq$ $d\left(R_{Z} \otimes_{R} M\right)+$ tr. $\operatorname{deg}_{k} Z$, where $d\left(R_{Z} \otimes M\right)$ is the Gelfand-Kirillov dimension of $R_{z} \otimes M$ viewed as an $R_{z}$-module and $R_{z}$ is viewed as a finitely generated $K$-algebra (not as a $k$-algebra). The result is primarily of a technical nature.

\section{Introduction}

1.1. $R$ will be a finitely generated algebra over a field $k$ of characteristic zero. Let $E$ be a central subring of $R$ containing $k$ with the property that every element of $E$ is regular in $R$. Let $R_{E}$ denote the localization of $R$ at $E$ (that is, $R_{E}$ is the ring obtained by inverting the elements of $E$ ). The ring $R_{E}$ will not generally be finitely generated as an algebra over $k$, but will be a finitely generated algebra over the field $K$ which arises as the quotient field of the integral domain $E$. Such a situation is typical if $R$ is a prime factor ring of an enveloping algebra of a finite dimensional Lie algebra, or a prime factor ring of the group algebra of a polycyclic-by-finite group.

If $M$ is an $R$-module it is natural to look at the Gelfand-Kirillov dimension of $M$ (denoted $d(M)$ ) over the field $k$ (that is, the vector spaces in the definition of $d(M)$ are $k$-vector spaces); we shall write $d_{R}(M)$ for the Gelfand-Kirillov dimension of the $R$-module $M$, viewing $R$ as an algebra over $k$. Put $S=R_{E}$; if $N$ is an $S$-module it is natural to look at $d(N)$ by viewing $N$ as an $S$-module and $S$ as an algebra over $K$; we shall write $d_{s}(N)$ for the Gelfand-Kirillov dimension of the $S$-module $N$, viewing $S$ as an algebra over $K$ (not as an algebra over $k$ ). 
So, for example, if $R=k[X, Y]$, the commutative polynomial ring in two indeterminates, and $E=k[X]$, then $S=k(X)[Y]$ and $d_{R}(R)=2$ while $d_{S}(S)=$ 1.

We determine the relationship between $d_{R}$ and $d_{S}$. More precisely, if $M$ is a finitely generated $R$-module such that $S \otimes_{R} M \neq 0$ we look at the relationship between $d_{R}(M)$ and $d_{S}\left(S \otimes_{R} M\right)$ (see Theorem 2.7 for a precise statement of the result).

The result is primarily of a technical nature. We give two examples towards the end of the paper to indicate how Theorem 2.7 may be applied. In [10], the example given in $\$ 3.3$ is used in a key lemma.

1.2. If $B$ is a finitely generated algebra over a field $k$, generated by a finite dimensional subspace $V$ containing $k$, and $M$ is a $B$-module generated by a finite dimensional subspace $M_{0}$, then the Gelfand-Kirillov dimension of $M$ is defined to be

$$
d_{B}(M)=\lim _{n \rightarrow \infty} \sup \frac{\log \operatorname{dim} V^{n} M_{0}}{\log n} .
$$

The reader is referred to [2], [5], [9] for details.

This dimension is most useful when applied to those algebras $B$ having the property that the associated graded algebra (determined by the filtration coming from the powers of $V$ viz. $\left.k=V^{0} \subset V^{1} \subset V^{2} \subset \cdots\right)$ is commutative. Such $B$ are called almost commutative algebras, and are precisely the factor rings of enveloping algebras. We include in our definition of almost commutative algebras the requirement that they are finitely generated.

\section{The localization theorem}

2.1. We begin with the assumption that $E$ is generated as an algebra over $k$ by the algebraically independent elements $z_{1}, \cdots, z_{n}$; that is $E=k\left[z_{1}, \cdots, z_{n}\right]$, the commutative polynomial ring in $n$ indeterminates. This assumption applies through to $\$ 2.6$. After dealing with this special case, the general case will be dealt with in $\$ 2.7$.

2.2. The ideas behind the following theorem are well known, see e.g. [8].

THEOREM. Let $x_{1}, \cdots, x_{n}$ be commuting indeterminates and let $T$ be the ring $R \otimes_{k} k\left(x_{1}, \cdots, x_{n}\right)$ where $k\left(x_{1}, \cdots, x_{n}\right)$ is the rational function field. Let $J$ be the left ideal of $T$ generated by the elements $x_{i}-z_{i}=1 \otimes x_{i}-z_{i} \otimes 1$ for $i=1, \cdots, n$. Then $T / J \cong S=R_{E}$ via the map 


$$
\phi\left(\sum_{1}^{m} a_{i} \otimes p_{i}\left(x_{1}, \cdots, x_{n}\right)\right)=\sum_{1}^{m} a_{i} p_{i}\left(z_{1}, \cdots, z_{n}\right)
$$

where $t^{-}$denotes the image of $t \in T$ in $T / J$. Moreover, $R$ embeds in $T / J$ through the embedding $R \rightarrow T$ defined by $r \rightarrow r \otimes 1$, and $\phi(R)$ is the natural copy of $R$ which lies in S. Finally, $\phi\left(k\left(x_{1}, \cdots, x_{n}\right)\right)=K$, the quotient field of $E$.

Proof. This is straightforward. The only point to be watched is that one really does require the $z_{1}, \cdots, z_{n}$ to be algebraically independent for $\phi$ to be well-defined as a map: $T / J \rightarrow R$.

The idea behind the proof of the localization theorem is to study $S$ by first obtaining information about $T$ and then making use of the isomorphism $T / J \cong S$.

2.3. We recall the definition of an $r$-sequence given in [6].

Definition. Let $B$ be an arbitrary ring, and $M$ a left $B$-module. A sequence of endomorphisms $\alpha_{1}, \cdots, \alpha_{n}$ in End ${ }_{B} M$ in called a regular endomorphism sequence on $M$, for brevity an $r$-sequence on $M$, if it satisfies the following conditions;

(i) $\alpha_{1} M+\cdots+\alpha_{n} M \neq M$

(ii) $\alpha_{k}\left(\alpha_{1} M+\cdots+\alpha_{k-1} M\right) \subset \alpha_{1} M+\cdots+\alpha_{k-1} M$ for $k>1$;

(iii) $\alpha_{1}^{-1}(0)=0 ; \alpha_{k}^{-1}\left(\alpha_{1} M+\cdots+\alpha_{k-1} M\right) \subset \alpha_{1} M+\cdots+\alpha_{k-1} M$ for $k>1$.

2.4. Proposition. Let $B$ be an almost commutative $k$-algebra, $M$ a finitely generated B-module, $\alpha_{1}, \cdots, \alpha_{t}$ an $r$-sequence on $M$. Put $\bar{M}=$ $M /\left(\alpha_{1} M+\cdots+\alpha_{t} M\right)$. Then $d_{B}(M) \geqq d_{B}(\bar{M})+t$.

Remark. The idea for this Proposition comes from [6, theorem 2.6] where the "same" result is proved with Krull dimension in place of Gelfand-Kirillov dimension. The "same" proof will work in both cases and so is omitted here. The only point to be watched is that one needs to use the fact that if $M$ is a finitely generated $B$-module and $d_{B}(M)=d$, then any chain of submodules $M=$ $M_{0} \supset M_{1} \supset \cdots$ with $d_{B}\left(M_{i} / M_{i+1}\right)=d$ for each $i$, must terminate after only finitely many terms (see e.g. [9, corollary 2.2]). At present, this result is only known to hold when $B$ is an almost commutative algebra, and it is for this reason that the proposition is restricted to such $B$. If one could extend this result to a larger class of rings then the main theorem of the present paper could be extended accordingly.

2.5. Proposition. Suppose $I$ is a left ideal of $R$ with $I \cap E=0$. Let $\rho_{i}$ be the image of $x_{i}-z_{i}$ in $\operatorname{End}_{T}(T / T I)$. Then $\rho_{1}, \cdots, \rho_{n}$ is an $r$-sequence on $T / T I$ (viewed as a $T$-module). 
Proof. Use $[7$, lemma 2.6] with appropriate changes of notation.

2.6. Proposition. Suppose $I$ is a left ideal of an almost commutative $k$ algebra $R$ with $I \cap E=0$. Then

$$
d_{R}(R / I) \geqq d_{S}(S / S I)+n .
$$

Proof. After Theorem 2.2, we have $S / S I \cong T /(T I+J)$ as $S$-modules, so $d_{s}(S / S I)=d_{T / J}(T /(T I+J))$ where $T / J$ is viewed as a finitely generated algebra over $k\left(x_{1}, \cdots, x_{n}\right)=\phi^{-1}(K)$. Of course, $d_{T / J}(T /(T I+J))=d_{T}(T /(T I+J))$. However, putting $M=T / T I$ and using the $r$-sequence $\rho_{1}, \cdots, \rho_{n}$ of the previous proposition, $\rho_{1} M+\cdots+\rho_{n} M=(T I+J) / T I$. So after Proposition 2.4 (with $B=$ $T), d_{T}(T / T I) \geqq d_{T}(T / T I+J)+n$; whence $d_{T}(T / T I) \geqq d_{S}(S / S I)+n$.

Notice that $T / T I \cong(R / I) \bigotimes_{k} k\left(x_{1}, \cdots, x_{n}\right)$ as vector spaces over $k\left(x_{1}, \cdots, x_{n}\right)$. This isomorphism will restrict to an isomorphism on the filtration subspaces of $T / T I$ and $R / I$ that are used to determine the Gelfand-Kirillov dimension of each of these modules (because a subset $\left\{v_{i}\right\}$ of $R / I$ is linearly independent over $k$ if and only if the images of these elements in $T / T I$ are linearly independent over $\left.k\left(x_{1}, \cdots, x_{n}\right)\right)$. Thus $d_{T}(T / T I)=d_{R}(R / I)$. This completes the proof.

2.7. We now drop the assumption that $E$ is a polynomial ring. Being an almost commutative algebra, $R$ has finite Gelfand-Kirillov dimension, hence so does $E$. This in turn implies that $K$, the quotient field of $E$, has finite transcendence degree over $k$. Consequently we can find algebraically independent elements $z_{1}, \cdots, z_{n}$ of $E$ with $n=$ tr. $\operatorname{deg}_{k} K=$ tr. $\operatorname{deg}_{k} E$. Put $F=$ $k\left[z_{1}, \cdots, z_{n}\right]$.

Let $M$ be a finitely generated $R$-module such that $S \otimes_{R} M \neq 0$ where, as before, $S=R_{E}$. There is a left ideal $I$ of $R$ with the properties:

(i) $R / I$ is isomorphic to a submodule of $M$;

(ii) $I \cap E=0$;

(iii) $d_{S}\left(S \otimes_{R} M\right)=d_{S}\left(S \otimes_{R}(R / I)\right)$.

Put $A=R_{F}$. It is clear from (ii) that $A \otimes_{R}(R / I) \neq 0$ and so by Proposition 2.6, $\quad d_{R}(M) \geqq d_{R}(R / I) \geqq d_{A}(A / A I)+n$. Notice that $S \otimes_{R}(R / I) \cong$ $S \otimes_{A}(A / A I)$, and $S$ may be considered as a localization of $A$ at its central subring generated by $E$ and $k\left(z_{1}, \cdots, z_{n}\right)$.

THEOREM. Let $R$ be an almost commutative $k$-algebra. Let tr. $\operatorname{deg}_{k} E=n$. If $M$ is a finitely generated $R$-module such that $S \otimes_{R} M \neq 0$, then

$$
d_{R}(M) \geqq d_{S}\left(S \otimes_{R} M\right)+n \text {. }
$$


Proof. On the basis of the notation introduced above, $d_{S}\left(S \otimes_{R} M\right)=$ $d_{S}\left(S \otimes_{A}(A / A I)\right)$ and so to prove the Theorem it is sufficient to show that $d_{S}\left(S \otimes_{A}(A / A I)\right) \leqq d_{A}(A / A I)$.

Let $N_{0}$ be a finite dimensional $k\left(z_{1}, \cdots, z_{n}\right)$-subspace of $N=A / A I$, which generates $N$ as an $A$-module. Let $K$ denote the quotient field of $E$. Then $K \otimes_{k\left(z_{1}, \cdots, z_{n}\right)} N_{0}$ is a finite dimensional $K$-subspace of $S \otimes_{A} N$ generating $S \otimes_{A} N$ as an $S$-module. Suppose $A=\bigcup_{n=0}^{x} V^{n}$ where $V$ is a finite dimensional $k\left(z_{1}, \cdots, z_{n}\right)$-subspace (containing $k\left(z_{1}, \cdots, z_{n}\right)$ ). Then

$$
\operatorname{dim}_{K}\left(K \otimes V^{n}\right)\left(K \otimes N_{0}\right)=\operatorname{dim}_{K}\left(K \otimes V^{n} N_{0}\right) \leqq \operatorname{dim}_{K\left(z_{1}, \cdots, z_{n}\right)} V^{n} N_{0}
$$

for all integers $n$, and so $d_{S}\left(S \otimes_{\mathrm{A}} N\right) \leqq d_{\mathrm{A}}(N)$.

2.8. The present approach does not make it easy to decide whether one actually obtains equality in the Theorem. Unfortunately we are unable to offer an example to show that the inequality may be strict.

It may be possible to extend Theorem 2.7 to apply to rings $R$ which arise as factor rings of group algebras of polycyclic-by-finite groups. In such cases the inequality may be strict. For example, take $R=M=K[G]$ where $G=$ $\left\langle x, y, z \mid x y x^{-1} y^{-1}=z\right\rangle$ and take $E=k\left[z, z^{-1}\right]$; then $d_{R}(M)=4$ and $d_{s}(S)=2$.

\section{Applications}

3.1. Let $R$ be a prime factor ring of the enveloping algebra, $U(\mathfrak{g})$, of a finite dimensional nilpotent Lie algebra $g$. Further assume that $k$ is algebraically closed. Let $E$ be the centre of $R$ and $K$ the quotient field of $E$. The structure of $R_{E}$ is well known [3]. The ring $R_{E}$ is isomorphic to a Weyl algebra $A_{m}(K)$, over the field $K$. The integer $m$ satisfies $d(R)=2 m+\operatorname{tr}$. $\operatorname{deg}_{k} K$. Put $S=R_{E}$.

If $I$ is a left ideal of $R$ with $I \cap E=0$ then $S \otimes_{R}(R / I) \neq 0$ and after [1], $d_{s}\left(S \otimes_{R}(R / I)\right) \geqq m$. Consequently Theorem 2.7 shows that $d_{R}(R / I) \geqq$ $m+\operatorname{tr} . \operatorname{deg}_{k} K$. It is a straightforward consequence of this that if $M$ is an $R$-module and $d_{R}(M)<\frac{1}{2}\left(d(R)+\operatorname{tr} . \operatorname{deg}_{k} K\right)$ then $\operatorname{ann}(M) E \neq 0$. In other words, one concludes that a faithful $R$-module must have Gelfand-Kirillov dimension at least $\frac{1}{2}\left(d(R)+\operatorname{tr} \cdot \operatorname{deg}_{k} K\right)$.

3.2. In [4] a generalization of Quillen's Lemma is proved: if $R$ is an almost commutative $k$-algebra, and $M$ a finitely generated $R$-module then $c\left(\operatorname{End}_{R} M\right) \leqq \mathrm{K}-\operatorname{dim} M$, where $c\left(\operatorname{End}_{R} M\right)$ denotes the maximal transcendence degree of a commutative subalgebra of $\operatorname{End}_{R} M$, and $\mathrm{K}$-dim $M$ is the Krull 
dimension of $M$. In particular, knowing the Krull dimension of $M$ enables one to bound $c\left(\operatorname{End}_{R} M\right)$. However, this bound is not always the best possible. For example, if $\mathfrak{a}$ is the 3-dimensional Heisenberg Lie algebra with basis $x, y, z$ and relations $[x, y]=z, z$ central, then K-dim $U(\mathfrak{a})=3$ but $c($ End $U(\mathfrak{a}))=c(U(\mathfrak{a}))$ $=2$.

We illustrate how it is possible by localizing and applying Theorem 2.7 to obtain a better bound for $c\left(\operatorname{End}_{R} M\right)$ in some situations.

3.3. Suppose $R$ and $E$ are as in 3.1 and use the notation there. Let $M$ be a left $R$-module with the property that every non-zero submodule $N$ of $M$ satisfies $S \otimes_{R} N \neq 0$. We shall obtain a bound for $c\left(\operatorname{End}_{R} M\right)$ which will usually be an improvement on the bound K-dim $M$ given by the generalization of Quillen's Lemma.

The condition on $M$ ensures that the natural map $\operatorname{End}_{R} M \rightarrow \operatorname{End}_{S}\left(S \otimes_{R} M\right)$ is an embedding. Let $C=k\left[\theta_{1}, \cdots, \theta_{r}\right]$ be a commutative subalgebra of $\operatorname{End}_{R} M$ such that

$$
c\left(\operatorname{End}_{R} M\right)=d(C)=\text { tr. } \operatorname{deg}_{k} C=r .
$$

Consider $C$ as a subring of $\operatorname{End}_{S}\left(S \otimes_{R} M\right)$. We may also consider $K$ as a subring of $\operatorname{End}_{S}\left(S \otimes_{R} M\right)$. Let $K[C]$ denote the (commutative) subring of $\operatorname{End}_{S}\left(S \otimes_{R} M\right)$ generated by $K$ and $C$. The generalization of Quillen's Lemma ensures that tr. $\operatorname{deg}_{K} K[C] \leqq \mathrm{K}-\operatorname{dim}_{S}\left(S \otimes_{R} M\right)$.

Now $S \cong A_{m}(K)$ and by [9, lemma 2.3], we have $K-\operatorname{dim}_{S}\left(S \otimes_{R} M\right) \leqq$ $d_{s}\left(S \otimes_{R} M\right)-m$. Hence, applying Theorem 2.7, tr. $\operatorname{deg}_{K} K[C] \leqq$ $d_{R}(M)-$ tr. $\operatorname{deg}_{k} K-m$.

Finally, we may assume without loss of generality that $C$ contained a transcendence basis for $K$ to begin with; this is because $E$ may be considered as a central subring of $\operatorname{End}_{R} M$, and $E$ contains a transcendence basis for $K$. Once this assumption is made it is clear that tr. $\operatorname{deg}_{k} C=\operatorname{tr} \cdot \operatorname{deg}_{k} K[C]+\operatorname{tr} \cdot \operatorname{deg}_{k} K$. Thus $c\left(\operatorname{End}_{R} M\right) \leqq d_{R}(M)-m$.

3.4. The restriction on $M$ in 3.3 is fairly natural. For any module $M$ let $t(M)$ denote the set of elements of $M$ annihilated by some non-zero element of $E$; $t(M)$ is in fact a submodule of $M$, the torsion submodule with respect to $E$, and $M / t(M)$ satisfies the restriction in 3.3 .

A typical example would be if $R=U(\mathfrak{a})$, where $a$ is the 3-dimensional Heisenberg algebra, and $M=R / R x$. The analysis in 3.3 yields $c\left(\operatorname{End}_{R} M\right) \leqq 1$ although $\mathrm{K}-\operatorname{dim} M=2$. In fact $\operatorname{End}_{R} M=k[\theta]$ where $\theta$ is the $R$-endomorphism given by $\theta(\overline{1})=z$. 


\section{REFERENCES}

1. I. N. Bernstein, The analytic continuation of generalized functions with respect to a parameter, Funkcional. Anal. i Prilozen 6 (1972), 26-40.

2. W. Bohro and H. Kraft, Uber die Gelfand-Kirillov Dimension, Math. Ann. 220 (1975), 201-210.

3. J. Dixmier, Enveloping Algebras, North-Holland Mathematical Library, 1977.

4. A. Joseph, A generalization of Quillen's Lemma and its application to the Weyl algebras, Isr. J. Math. 28 (1977), 177-192.

5. A. Joseph, Dimension en algèbre non-commutatif, Lecture Notes at Univ. Paris, 1980.

6. R. Resco, Transcendental division algebras and simple noetherian rings, Isr. J. Math. 32 (1979), 236-256.

7. R. Resco, Krull dimension of noetherian algebras and extensions of the base field, Commun. Algebra 8 (1980), 161-183.

8. J. E. Roos, Complements à l'étude des quotients primitifs des algèbres envéloppantes des algèbres de Lie semi-simples, C.R. Acad. Sci. Paris 276 (1973), 447-450.

9. S. P. Smith, Krull dimension of the enveloping algebra of $\mathrm{sl}(2, C)$, J. Algebra 71 (1981), 189-194.

10. S. P. Smith, Krull dimension of factor rings of enveloping algebras of semi-simple Lie algebras, Proc. Camb. Phil. Soc., to appear.

Department of Mathematics

UNIVERSITY OF SOUTHERN CALIFornia

Los ANGELES, CA 90089 USA

Present Address:

Department of Mathematics

UNIVERSITY OF WARWICK

COVENTRY CV4 7AL, ENGLAND 